\title{
Identifying AGN Balmer absorptions and stratified narrow emission-line region kinematics in SDSS J112611.63+425246.4 (Research Note)
}

\author{
J. Wang and D. W. Xu
}

\author{
National Astronomical Observatories, Chinese Academy of Sciences, 100012 Beijing, PR China \\ e-mail: wj@nao.cas.cn
}

Received 22 August 2014 / Accepted 21 October 2014

\begin{abstract}
Context. Balmer absorption is a rare phenomenon in active galactic nuclei (AGNs). So far, only seven Balmer-absorption AGNs have been reported in the literature.

Aims. We here report the identification of SDSS J112611.63+425246 as a new Balmer-absorption AGN through our spectral analysis and study the kinematics of its narrow emission-line region (NLR).

Methods. We modeled the continuum by a linear combination of a starlight component, a power law from the central AGN, and the emission from the FeII complex. After subtracting the modeled continuum, each emission or absorption line profile is a sum of multi-Gaussian functions. All the line shifts were determined with respect to the modeled starlight component.

Results. By using the host starlight as a reference for the local system, both $\mathrm{H} \alpha$ and $\mathrm{H} \beta$ show AGN absorptions with a blueshift of $\sim 300 \mathrm{~km} \mathrm{~s}^{-1}$. We identify a strong anticorrelation between the inferred velocity shifts and the ionization potential for various narrow emission lines, which suggests a stratified NLR kinematics. A de-accelerated outflow is implied for the inner NLR gas, an accelerated inflow for the outer NLR gas. This complicated NLR kinematics additionally implies that AGN narrow emission lines, even for the low-ionized lines, might not be a reliable substitute for the velocity of the local system.
\end{abstract}

Key words. galaxies: active - galaxies: peculiar - galaxies: individual: SDSS J112611.63+425246.4

\section{Introduction}

The feedback from a central active galactic nucleus (AGN) is now believed to be a potential key ingredient in understanding the coevolution of the AGN and its host galaxy. A widely accepted scenario is that the growth of a supermassive black hole (SMBH) regulates host star formation by sweeping out circumnuclear gas (e.g., Silk \& Rees 1998; Somerville et al. 2008; Hirschmann et al. 2013; Di Matteo et al. 2007; Fabian 1999; Granato et al. 2004; Croton et al. 2006).

The evidence of outflow from an AGN on various scales has been identified in multiwavelength bands from radio to X-ray (see Veilleux et al. 2005 and Fabian 2012 for reviews). AGN outflow has been diagnosed by the blueshifted absorption lines. Although the narrow absorption lines with width $\leq 500 \mathrm{~km} \mathrm{~s}^{-1}$ are frequently identified in type I AGNs in UV and X-ray ( 50\%, e.g., Hamann \& Sabra 2004), Balmer-absorption AGNs are still rare cases. So far, only seven Balmer-absorption AGNs are reported in the literature. They are NGC 4151 (Hutching et al. 2002), SDSS J0839+3805 (Aoki et al. 2006), SDSS J1259+1213 (Hall 2007), SDSS J1029+4500 (Wang et al. 2008), SDSS 1723+5553 (Aoki 2010), LBQS 1206+1052 (Ji et al. 2012), and SDSS J2220+0109 (Ji et al. 2013). Because of their rarity, the identification of more Balmer-absorption AGNs is essential for subsequently studying the nature of the AGN Balmer absorption-line region (BAR). First, recent studies pointed out that rigorous conditions with a high hydrogen column density of $\sim 10^{21-22} \mathrm{~cm}^{-2}$ are required to excite neutral hydrogen atoms to $n=2$ shell by Ly $\alpha$ resonant pumping (e.g., Ji et al. 2012). Second, the Balmer absorption lines can be used as a diagnostic for the kinematics of the natural gas around central AGNs.

In this paper, we report a detailed spectroscopic analysis for SDSS J112611.63+425246.4 (hereafter SDSS J1126+4252 for short $)^{1}$, which allows us to identify the object as a new Balmerabsorption AGN and to identify a stratified kinematics in its narrow emission-line region (NLR) with respect to the systematic velocity determined from the host galaxy.

\section{Spectral analysis}

The optical spectrum of SDSS J1126+4252 was extracted when we carried out a systematic X-ray and optical spectral analysis on the XMM-Newton 2XMMi/SDSS-DR7 catalog that was originally cross-matched by Pineau et al. (2011). The catalog contains a total of more than $30000 \mathrm{X}$-ray point-like sources (with an X-ray position accuracy $\leq 5^{\prime \prime}$ ) that have an SDSS-DR7 optical counterpart with an identification probability higher than $90 \%$. The spectrum of the object was taken by the SDSS dedicated $2.5 \mathrm{~m}$ wide-field telescope on February 27, 2004.

The one-dimensional spectrum of the object was analyzed with the IRAF $^{2}$ package, including Galactic extinction

\footnotetext{
1 This object has been analyzed by Hu et al. (2008) in their large type I AGN sample. The Balmer absorptions and starlight component were, however, not taken into account in their spectral modeling.

2 IRAF is distributed by the National Optical Astronomy Observatory, which is operated by the Association of Universities for Research in Astronomy, Inc., under cooperative agreement with the National Science Foundation.
} 


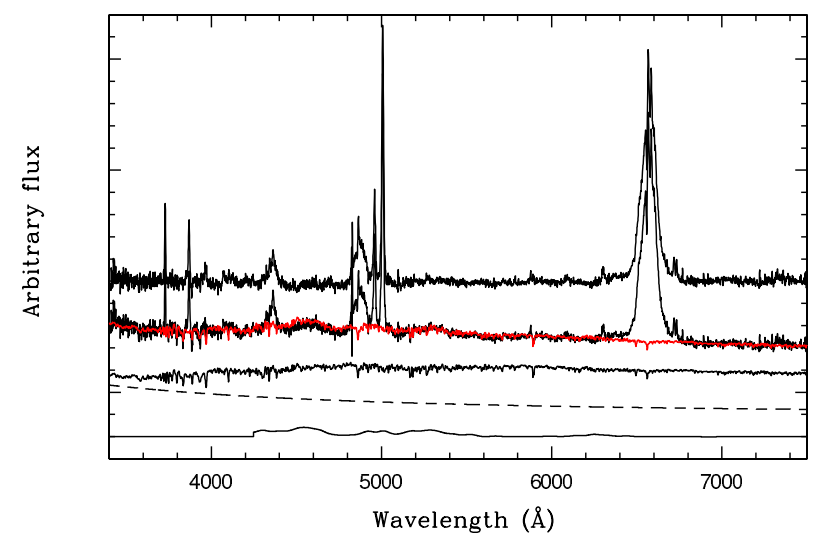

Fig. 1. Illustration of the modeling and removal of the continuum. The continuum-subtracted emission-line spectrum is shown by the top curve. Below the emission-line spectrum, the modeled continuum is overplotted by the red curve on the observed spectrum. The modeled continuum is obtained by a linear combination of a starlight component, a power law continuum from the AGN, and the emission from the FeII complex, which are displayed in ordinals below the observed spectrum. All the spectra are shifted vertically by an arbitrary amount for visibility.

correction, transformation to the rest frame, starlight component removal, and emission and absorption line profiling. We at first corrected the Galactic extinction for the color excess $E(B-V)$ taken from the Galactic reddening map of Schlegel, Finkbeiner, and Davies (Schlegel et al. 1998), by assuming an $R_{V}=3.1 \mathrm{ex}-$ tinction law of the Milky Way (Cardelli et al. 1989). The spectrum was then de-redshifted to its rest frame, along with the flux correction due to the relativity effect basing upon the measured redshift provided by the SDSS pipelines. The object has a nominal redshift of $z=0.15592 \pm 0.00121$, which corresponds to a velocity uncertainty of $363 \mathrm{~km} \mathrm{~s}^{-1}$.

The total light spectrum at the rest frame is displayed in Fig. 1. It shows that there is significant contamination from its host galaxy. To isolate the AGN emission-line spectrum, we modeled the continuum by a linear combination of a power law continuum, an FeII complex template, and the seven eigenspectra of starlight. The adopted FeII template was taken from Boroson \& Green (1992). The eigenspectra were built from the standard single stellar population spectral library developed by Bruzual \& Charlot (2003) through the principal component analysis (PCA) method (e.g., Wang \& Wei 2008; Francis et al. 1992). An additional Galactic extinction curve with $R_{V}=3.1$ was included in the modeling to account for the intrinsic extinction of the host galaxy. $\chi^{2}$ minimizations were iteratively performed over the rest-frame wavelength range from 3700 to $7000 \AA$, except for the regions with strong emission lines. The line width of the FeII template and the velocity dispersion of the starlight were predetermined through cross-correlation method before each iteration. The removal of the continuum is illustrated in Fig. 1 as well.

The AGN emission and absorption lines were subsequently modeled on the isolated line spectrum by using the SPECFIT task (Kriss 1994) in the IRAF package. The line modelings are schematically presented in the left and right panels in Fig. 2 for the $\mathrm{H} \alpha$ and $\mathrm{H} \beta$ regions. The two narrow Balmer absorptions are marked by the vertical short lines in the figure. Each emission line is modeled by a free Gaussian function, except for the broad $\mathrm{H} \alpha$ emission and the [OIII] doublet. Figure 2 clearly shows that a linear combination of two broad $\mathrm{H} \alpha$ components is required to

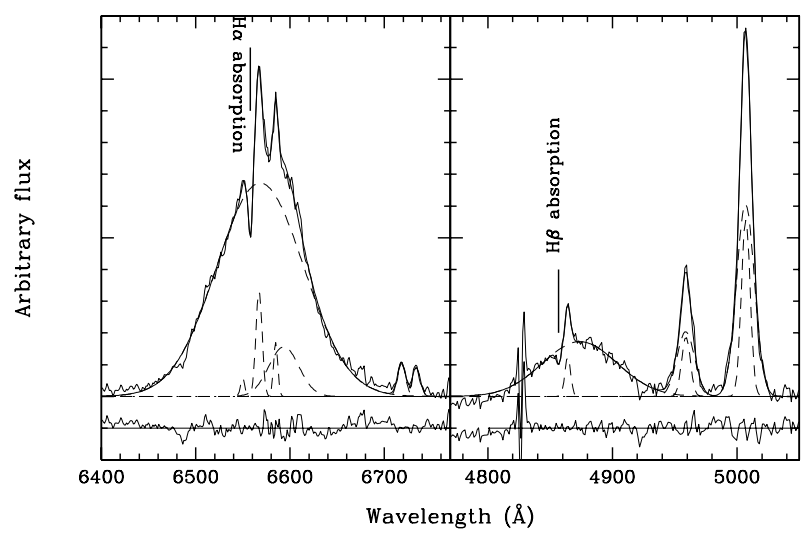

Fig. 2. Line profile modelings by a sum of a set of Gaussian components for the $\mathrm{H} \alpha$ (left panel) and $\mathrm{H} \beta$ (right panel) regions. The blueshifted Balmer absorptions are marked by the short vertical lines. In each panel, the observed and modeled line profiles are plotted by light and heavy solid lines. Each Gaussian component in emission is shown by a dashed line. The subpanel underneath each line spectrum presents the residuals between the observed and modeled profiles.

properly reproduce the observed line profile. We measured the FWMH of the total broad $\mathrm{H} \alpha$ emission with the IRAF/SPLOT task from the residual profile that was obtained by subtracting the modeled narrow emission (i.e., $\mathrm{H} \alpha$, [NII] $\lambda \lambda 6548,6583$ ) and absorption lines from the the observed profile. In addition to the narrow peak, a broad and blueshifted component is necessary to model the small blue wing of the [OIII] $\lambda 5007$ pro-

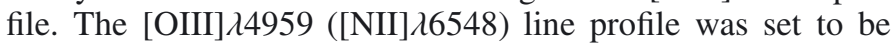
the same as $[\mathrm{OIII}] \lambda 5007$ ([NII] $\lambda 6583)$. The intensity ratio of the [OIII] ([NII]) doublet was fixed to the theoretical value of 3 . In total, the freedom in the $\chi^{2}$ minimization is 21 and 15 for the $\mathrm{H} \alpha$ and $\mathrm{H} \beta$ regions.

\section{Results and discussions}

The measured line properties are tabulated in Table 1. The reported flux of the $\mathrm{H} \alpha$ broad emission (and the [OIII] $\lambda 5007$ line emission) is the sum of the two fitted components. The quoted line width and velocity shift is based on the fitted narrow peak for the [OIII] line. The flux of the FeII blends (FeII 14570 ) was measured in the rest-frame wavelength range from 4434 to $4684 \AA$, which results in a parameter of RFe of $0.64 \pm 0.20$. RFe is defined as the flux ratio between the FeII $\lambda 4570$ and $\mathrm{H} \beta$ broad component. All the reported line widths were not corrected for the intrinsic instrument resolution of $\sigma_{\text {inst }} \approx 65 \mathrm{~km} \mathrm{~s}^{-1}$. Because of the evident contamination of the starlight in the integrated spectrum, we calculated the reported line shifts with respect to the modeled starlight component ${ }^{3}: \Delta v=\Delta v_{\text {line }}-\Delta v_{\text {host }}$, where $\Delta v_{\text {line }}$ and $\Delta v_{\text {host }}$ are the modeled velocity shifts with respect to the nominal redshift for a given emission or absorption line and for the host galaxy. A negative value of $\Delta v$ corresponds to a blueshift, a positive value to a redshift.

All the uncertainties reported in the table (except for the FWHM of $\mathrm{H} \alpha_{\mathrm{b}}$ ) only include the errors at $1 \sigma$ significance level resulting from the $\chi^{2}$ minimizations. The error of the FWHM of $\mathrm{H} \alpha_{\mathrm{b}}$ was obtained from a statistics on the multiple measurements

3 Hu et al. (2008) used the [OIII] $\lambda 5007$ line as a reference and found that the [OII] emission line might be a more reliable reference than either [OIII] or $\mathrm{H} \beta$. Our results are consistent with their measurements if the [OIII] line is used as a reference. 
by the IRAF/SPLOT task. A proper error propagation was taken into account in the reported uncertainties of $\Delta v$.

\subsection{Balmer absorption lines}

We argue that the observed blueshifted Balmer absorption lines most likely result from an outflow from the central engine, and not from the host galaxy. The starlight component was properly removed from the observed integral spectrum, as described above. Moreover, the lack of a strong Balmer break enabled us to exclude that the observed Balmer absorptions originate from a post-starburst galaxy with strong Balmer absorptions (e.g., Brotherton et al. 1999; Wang \& Wei 2006). In interpreting of the Balmer absorptions in AGNs, the intrinsic equivalent width (EW) depends on whether the absorbing gas covers the BLR or not (e.g., de Kool et al. 2001). The latter scenario is favored in the object from comparing the measured Balmer absorption EW ratio with its theoretical value. In an absorption line without saturation, its EW could be related to its column density $N$ as (Jenkins 1986)

$E W_{\lambda}=\frac{\pi e^{2} f \lambda^{2} N}{m_{\mathrm{e}} c^{2}}$,

where $f$ is the oscillator strength. A theoretical $f \lambda$ value of 7.26 is therefore expected for the $\mathrm{H} \alpha$-to- $\mathrm{H} \beta$ ratio, which is very close to the observed EW ratio of $E W(\mathrm{H} \alpha) / E W(\mathrm{H} \beta)=$ $7.38 \pm 4.32$ when both absorption lines are normalized with respect to the modeled AGN continuum. In contrast, the observed ratio is closed to 1 if both absorption lines are normalized to the corresponding broad emission line. This comparison therefore indicates that the absorbing gas responsible for the Balmer transitions is not saturated and fully covers the continuum source.

We furthermore estimated the neutral hydrogen column density from Eq. (1). The inferred column densities of hydrogen at $n=2$ shell from the $\mathrm{H} \alpha$ and $\mathrm{H} \beta$ absorption lines are $N_{\mathrm{HI}, 2}=$ $(1.2 \pm 0.5) \times 10^{14} \mathrm{~cm}^{-2}$ and $(1.6 \pm 2.2) \times 10^{14} \mathrm{~cm}^{-2}$. The neutral hydrogen column density was derived from $N_{\mathrm{HI}} \approx N_{\mathrm{HI}, 1}+N_{\mathrm{HI}, 2}$, where $N_{\mathrm{HI}, 1}$ is the column density of hydrogen at $n=1$ shell and is estimated by following Hall (2007):

$\frac{N_{\mathrm{HI}, 1}}{N_{\mathrm{HI}, 2}}=\frac{1}{4 \tau_{\mathrm{Ly} \alpha}} \mathrm{e}^{\frac{10.2 \mathrm{eV}}{k T}}$,

where $\tau_{\mathrm{Ly} \alpha}$ is the optical depth at the center of the Ly $\alpha$ absorption. The depth $\tau_{\mathrm{Ly} \alpha}$ was inferred from the relationship $\tau_{\mathrm{Ly} \alpha}=$ $0.12 \tau_{\mathrm{H} \alpha}\left(N_{\mathrm{HI}, 1} / N_{\mathrm{HI}, 2}\right)$ (see Eq. (1) in Aoki 2010). Substituting this relationship into Eq. (2) results in a relation

$\frac{N_{\mathrm{HI}, 1}}{N_{\mathrm{HI}, 2}}=\frac{1.44}{\sqrt{\tau_{\mathrm{H} \alpha}}} \mathrm{e}^{\frac{5.1 \mathrm{eV}}{k T}}$.

Taking $T=7500 \mathrm{~K}$ (Osterbrock \& Ferland 2006) and $\tau_{\mathrm{H} \alpha}=$ $1.69 \times 10^{5} E W(\mathrm{H} \alpha) / \lambda / b=8.66$ (where $b$ is the Doppler parameter of the absorption line after correcting for the intrinsic instrumental resolution), the inferred neutral hydrogen column density is $\sim 1.5 \times 10^{17} \mathrm{~cm}^{-2}$.

\subsection{UV and $X$-ray observations}

SDSS J1126+4252 is particularly weak and hard in UV and $\mathrm{X}$-ray bands. The object is a common source in the second $X M M-N e w t o n$ serendipitous source catalog (XMMSSC) and the XMM-Newton optical Monitor serendipitous UV source survey catalog (XMMOMSUSS). Vagnetti et al. (2010) showed that the inferred specific luminosities at $2500 \AA$ and $2 \mathrm{keV}$ are $4.8 \times 10^{28} \mathrm{erg} \mathrm{s}^{-1} \mathrm{~Hz}^{-1}$ and $6.6 \times 10^{23} \mathrm{erg} \mathrm{s}^{-1} \mathrm{~Hz}^{-1}$. Its very hard $\mathrm{X}$-ray spectrum can additionally be derived from the very high hardness ratios ${ }^{4}: \mathrm{HR} 3=0.52$ and $\mathrm{HR} 4=0.63$.

Ji et al. $(2012,2013)$ recently pointed out that a rigorous condition is required for the formation of Balmer absorptions. The absorptions are likely caused by Ly $\alpha$ resonant pumping in a partially ionized region with a high column density of $N_{\mathrm{H}} \sim 10^{21-22} \mathrm{~cm}^{-2}$. A heavy obscuration as a result of the required high column density is a possible explanation of the observed extremely weak and hard emission in both UV and X-ray.

\subsection{Eddington ratio and $S M B H$ mass}

We estimated the SMBH mass $M_{\mathrm{BH}}$ in terms of its $\mathrm{H} \alpha$ broad component according to the calibration in Greene \& Ho (2007):

$$
\begin{aligned}
M_{\mathrm{BH}}= & 3.0_{-0.5}^{+0.6} \times 10^{6}\left(\frac{L_{\mathrm{H} \alpha}}{10^{42} \mathrm{erg} \mathrm{s}^{-1}}\right)^{0.45 \pm 0.03} \\
& \times\left(\frac{F W H M_{\mathrm{H} \alpha}}{1000 \mathrm{~km} \mathrm{~s}^{-1}}\right)^{2.06 \pm 0.06} M_{\odot}
\end{aligned}
$$

where $L_{\mathrm{H} \alpha}$ is the intrinsic luminosity of the $\mathrm{H} \alpha$ broad component corrected for the local extinction. The extinction was inferred from the narrow-line ratio $\mathrm{H} \alpha / \mathrm{H} \beta$, assuming the Balmer decrement for standard case $\mathrm{B}$ recombination and a Galactic extinction curve with $R_{V}=3.1$. With the estimated $M_{\mathrm{BH}}$, the Eddington ratio $L / L_{\text {Edd }}$ (where $L_{\text {Edd }}=1.26 \times 10^{38} M_{\mathrm{BH}} / M_{\odot}$ is the Eddington luminosity) was obtained from a combination of the bolometric correction of $L_{\mathrm{bol}}=9 \lambda L_{\lambda}(5100 \AA$ ) (Kaspi et al. 2000) and the $L_{5100 \AA}-L_{\mathrm{H} \alpha}$ relation reported in Greene \& Ho (2005),

$\lambda L_{\lambda}(5100 \AA)=2.4 \times 10^{43}\left(\frac{L_{\mathrm{H} \alpha}}{10^{42} \mathrm{erg} \mathrm{s}^{-1}}\right)^{0.86} \mathrm{erg} \mathrm{s}^{-1}$.

This luminosity relation has an rms scatter of 0.2 dex around the best-fit line. The calculated $M_{\mathrm{BH}}$ and $L / L_{\mathrm{Edd}}$ are $\approx 1.8 \times 10^{8} M_{\odot}$ and $\approx 0.06$. By combining the intrinsic scatters of the relationships and the uncertainties derived from our line modelings, a proper error propagation returns $1 \sigma$ uncertainties of 0.40 dex and 0.45 dex for the calculated $M_{\mathrm{BH}}$ and $L / L_{\mathrm{Edd}}$.

We argue that the inferred $M_{\mathrm{BH}}$ from the broad $\mathrm{H} \alpha$ emission agrees with the properties of the host galaxy. With the velocity dispersion of the host galaxy of $\sigma_{\star} \sim 270 \mathrm{~km} \mathrm{~s}^{-1} \mathrm{ob}-$ tained from our continuum modeling, the $M_{\mathrm{BH}}-\sigma_{\star}$ relation of $\log \left(M_{\mathrm{BH}} / M_{\odot}\right)=8.13+4.02 \log \left(\sigma_{\star} / 200 \mathrm{~km} \mathrm{~s}^{-1}\right.$ ) (Tremaine et al. 2004) yields a blackhole mass of $\log \left(M_{\mathrm{BH}} / M_{\odot}\right) \sim 8.7$, which is highly consistent with the value estimated from the broad $\mathrm{H} \alpha$ emission.

\subsection{Stratified NLR kinematics}

The spectral analysis allows us to study the line shifts in SDSS J1126+4252 by using its host starlight component as a reference of the systematic velocity. One can see from Table 2 that all the low-ionized narrow emission lines show a redshift with respect to its host galaxy, while a blueshift is identified in the high-ionized emission line [NeIII] $\lambda 3868$. It is interesting

\footnotetext{
4 The hardness ratios are defined as HR3 $=(\mathrm{CR} 4-\mathrm{CR} 3) /(\mathrm{CR} 4+\mathrm{CR} 3)$ and HR4 $=(\mathrm{CR} 5-\mathrm{CR} 4) /(\mathrm{CR} 5+\mathrm{CR} 4)$, where CR3, CR4, and CR5 are the count rates in the energy bands $1-2,2-4.5$, and $4.5-12 \mathrm{keV}$, respectively.
} 
that the $[\mathrm{OIII}] \lambda 5007$ emission line has a marginal blueshift of $\Delta v=-10 \pm 30 \mathrm{~km} \mathrm{~s}^{-1}$.

A strong anticorrelation between the velocity shifts and ionization potential (IP) is shown in Fig. 3. The velocity shift of the emitting gas of neutral hydrogen atom is taken from the measurement of narrow $\mathrm{H} \alpha$ emission, both because the narrow $\mathrm{H} \alpha$ and $\mathrm{H} \beta$ emission show comparable velocity shifts and because of the higher signal-to-noise ratio of the narrow $\mathrm{H} \alpha$ line. An average value of velocity shift is adopted in the figure for the [SII] doublet. The best fit yields a relation of $\Delta v=(186.4 \pm$ $35.4)-(4.00 \pm 0.80)$ IP. Komossa et al. (2008) proposed a similar correlation between the line shift and IP in the narrowline Seyfert 1 galaxies with a high [OIII] line blueshift over $150 \mathrm{~km} \mathrm{~s}^{-1}$.

Because the AGN NLR gas is believed to be generally stratified in density and ionization potential (e.g., Filippenko \& Halpern 1984; Filippenko 1985; De Robertis \& Osterbrock 1986), our fitted relationship implies a complicated NLR kinematics in SDSS J1126+4252. A de-accelerated outflow is expected for the inner NLR gas, while an accelerated inflow for the outer NLR gas. The turnover of the radial velocity occurs at the [OIII] $\lambda 5007$ emission-line gas, whose radial velocity shift with respect to the local system determined from the host starlight is very close to zero. Although the outflows from central AGNs on various scales have frequently been identified in AGNs (e.g., Komossa et al. 2008 and Fabian 2012 for a recent review), the inflows have already been revealed in a few of nearby AGNs through integrated field spectroscopic observations in both optical band and near-infrared (e.g., Fathi et al. 2005; Storchi-Bergmann et al. 2007; Riffel et al. 2008, 2013; Riffel \& Storchi-Bergmann 2011). The observations reveal distinct kinematics for different emitting gas. The inflowing gas to the central active nucleus can be traced by the $\mathrm{H}_{2}$ emission, the outflowing gas by the [FeII] emission, which is similar to the kinematics revealed in SDSS J1126+4252.

The implications described above are based on the scenario in which the detected NLR lines are seen in front of the central AGN. We cannot exclude an alternative scenario here in which these NLR lines are seen behind the central source. In this scenario, the obtained relationship implies a de-accelerated inflow for the inner NLR gas, and an accelerated outflow for the outer NLR gas.

Our spectral analysis indicates that in SDSS J1126+4252 all the narrow emission lines, except for the [OIII], show strong velocity shifts with respect to the local system determined from the host starlight. This causes concern that AGN narrow emission lines, even for the low-ionized lines, might not be a reliable substitute for the velocity of the local system. A large sample is needed to perform a more detailed examination of the relationship between the velocity shifts of various narrow emission lines and the local system determined from host starlight in the future.

\section{Conclusions}

We performed a detailed spectral analysis on SDSS J11261.63+425246.4, which allowed us to identify the object as a new Balmer-absorption AGN. By using the modeled host starlight as the reference of the local system, a stratified kinematics was identified in the NLR of the object, that is, a strong anticorrelation between the inferred velocity shifts and ionization potentials. The revealed relationship implies a de-accelerated outflow stream for its inner NLR gas, and an accelerated inflow stream for its outer NLR gas.

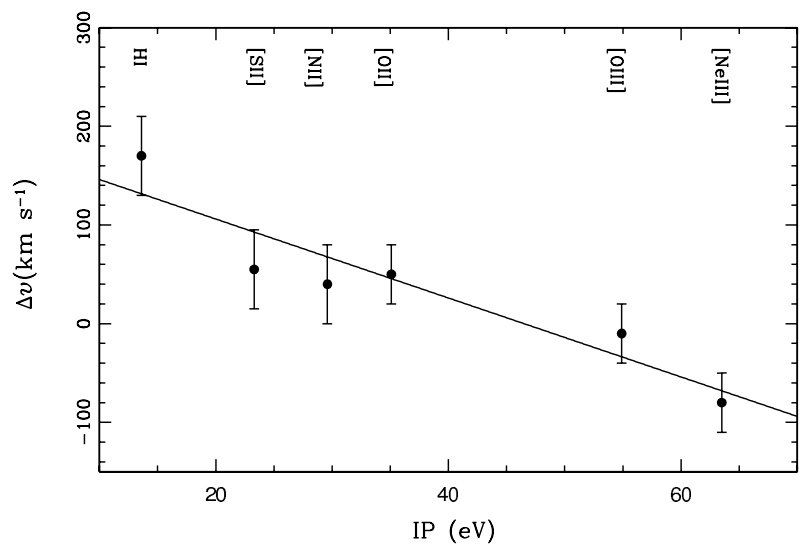

Fig. 3. Correlation between velocity shifts and ionization potential (IP). A positive velocity denotes a redshift, a negative value a blueshift. The best-fit line is overplotted by the solid line.

Table 1. Spectral properties of SDSS J1126+4252.

\begin{tabular}{lccc}
\hline \hline \multicolumn{4}{c}{ Emission } \\
\hline Line & $\begin{array}{c}\text { Flux } \\
\mathrm{erg} \mathrm{s}^{-1} \mathrm{~cm}^{-2} \\
(2)\end{array}$ & $\begin{array}{c}F W H M \\
\mathrm{~km} \mathrm{~s}^{-1} \\
(3)\end{array}$ & $\begin{array}{c}\Delta v \\
\mathrm{~km} \mathrm{~s}^{-1} \\
(4)\end{array}$ \\
\hline$[\mathrm{OII}] \lambda 3727$. & $2.3 \pm 0.2$ & $520 \pm 40$ & $50 \pm 30$ \\
{$[\mathrm{NeIII}] \lambda 3868$} & $2.6 \pm 0.2$ & $710 \pm 50$ & $-80 \pm 30$ \\
$\mathrm{FeII} \lambda 4570 \ldots$ & $8.3 \pm 0.3$ & $\sim 3 \times 10^{3}$ & $100 \pm 150$ \\
$\mathrm{H} \beta_{n} \ldots \ldots \ldots$ & $0.7 \pm 0.5$ & $340 \pm 250$ & $150 \pm 80$ \\
$\mathrm{H} \beta_{b} \ldots \ldots \ldots$. & $13.0 \pm 1.1$ & $4350 \pm 230$ & $750 \pm 30$ \\
{$[\mathrm{OIII}] \lambda 5007$.} & $15.8 \pm 2.9$ & $500 \pm 90$ & $-10 \pm 30$ \\
$\mathrm{H} \alpha_{n} \ldots \ldots \ldots$ & $2.7 \pm 1.1$ & $350 \pm 110$ & $170 \pm 40$ \\
$\mathrm{H} \alpha_{b} \ldots \ldots \ldots$ & $84.3 \pm 2.8$ & $4640.0 \pm 70$ & $\ldots \ldots$. \\
{$[\mathrm{NII}] \lambda 6583$.} & $1.0 \pm 0.6$ & $240 \pm 120$ & $40 \pm 40$ \\
{$[\mathrm{SIII}] \lambda 6716 \ldots$} & $1.1 \pm 0.1$ & $440 \pm 40$ & $50 \pm 30$ \\
{$[\mathrm{SII}] \lambda 6731 \ldots$} & $1.0 \pm 0.1$ & $440 \pm 40$ & $60 \pm 30$ \\
\hline \multicolumn{4}{c}{ Absorption } \\
\hline $\mathrm{H} \beta \ldots \ldots \ldots$ & $28.8 \pm 11.1$ & $290 \pm 540$ & $-300 \pm 70$ \\
$\mathrm{H} \alpha \ldots \ldots \ldots$ & $3.9 \pm 5.6$ & $210 \pm 70$ & $-250 \pm 40$ \\
\hline
\end{tabular}

Acknowledgements. The authors thank Todd A. Boroson and Richard F. Green for providing the optical FeII template. This research has made use of the SDSS archive data that are created and distributed by the Alfred P. Sloan Foundation. This study is supported by the Chinese National Basic Research Program (973 program, Grant No. 2014CB845800). J.W. is supported by the National Natural Science Foundation of China (grant No. 11473036). D.W.X. is supported by the National Natural Science Foundation of China under grant No. 11273027.

\section{References}

Aoki, K. 2010, PASJ, 62, 1333

Aoki, K., Iwata, I., Ohta, K., et al. 2006, ApJ, 651, 84

Boroson, T. A., \& Green, R. F. 1992, ApJS, 80, 109

Brotherton, M. S., van Breugel, W., Stanford, S. A., et al. 1999, ApJ, 520, 87

Bruzual, G., \& Charlot, S. 2003, MNRAS, 344, 1000

Cardelli, J. A., Clayton, G. C., \& Mathis, J. S. 1989, ApJ, 345, 245

Croton, D. J., Springel, V., White, S. D. M., et al. 2006, MNRAS, 365, 11

de Kool, M., Arav, N., Becker, R. H., et al. 2001, ApJ, 548, 609

De Robertis, M. M., \& Osterbrock, D. E. 1986, ApJ, 301, 727

Di Matteo, P., Combes, F., Melchior, A.-L., \& Semelin, B. 2007, A\&A, 468, 61

Fabian, A. C. 1999, MNRAS, 308, L39

Fabian, A. C. 2012, ARA\&A, 50, 455

Fathi, K., Storchi-Bergmann, T., Riffel, R. A., et al. 2006, ApJ, 641, L25

Filippenko, A. V. 1985, ApJ, 289, 475

Filippenko, A. V., \& Halpern, J. P. 1984, ApJ, 285, 458

Francis, P. J., Hewett, P. C., Foltz, C. B., \& Chaffee, F. H. 1992, ApJ, 398, 476

Granato, G. L., De Zotti, G., Silva, L., Bressan, A., \& Danese, L. 2004, ApJ, 600,580 
J. Wang \& D. W. Xu: Balmer-absorptions and stratified NLR kinematics in SDSS J112611.63+425246.4 (RN)

Greene, J. E. \& Ho, L. C. 2005, ApJ, 630, 122

Greene, J. E., \& Ho, L. C. 2007, ApJ, 670, 92

Hall, P. B. 2007, AJ, 133, 1271

Hamann, F., \& Sabra, B. 2004, ASPC, 311, 203

Hirschmann, M., Naab, T., Davé, R., et al. 2013, MNRAS, 436, 2929

Hu, C., Wang, J. M., Ho, L. C., et al. 2008, ApJ, 687, 78

Hutchings, J. B., Crenshaw, D. M., Kraemer, S. B., et al. 2002, AJ, 124, 2543

Jenkins, E. B. 1986, ApJ, 304, 739

Ji, T., Wang, T.-G., Zhou, H.-Y., \& Wang, H.-Y. 2012, RAA, 12, 369

Ji, T., Zhou, H.-Y., Wang, T.-G., \& Wang, H.-Y. 2013, ChA\&A, 37, 17

Kaspi, S., Smith, P. S., Netzer, H., et al. 2000, ApJ, 533, 631

Komossa, S., Xu, D., Zhou, H., \& Storchi-Bergmann, T. 2008, ApJ, 680, 926

Kriss, G. 1994, Astronomical Data Analysis Software and systems III, ASP Conf. Ser., 61, 437

Osterbrock, D. E., \& Ferland, G. J. 2006, Astrophysics of Gaseous Nebulae and Active Galactic Nuclei, 2nd edn. (University Science Books)
Pineau, F.-X., Motch, C., Carrera, F. et al. 2011, A\&A, 527, 126 Riffel, R. A., \& Storchi-Bergmann, T. 2011, MNRAS, 417, 2752

Riffel, R. A., Storchi-Bergmann, T., Winge, C., et al. 2008, MNRAS, 385, 1129

Riffel, R. A., Storchi-Bergmann, T., \& Winge, C. 2013, MNRAS, 430, 2249

Schlegel, D., Finkbeiner, D. P., \& Davis, M. 1998, ApJ, 500, 525

Silk, J., \& Rees, M. J. 1998, A\&A, 331, L1

Somerville, R. S., Hopkins, P. F., Cox, T. J., Robertson, B. E., \& Hernquist, L. 2008, MNRAS, 391, 481

Storchi-Bergmann, T., Dors, O. L., Jr., Riffel, R. A., et al. 2007, ApJ, 670, 959 Tremaine, C. A., Heckman, T. M., Kauffmann, G., et al. 2004, ApJ, 613, 898

Vagnetti, F., Turriziani, S., Trevese, D., \& Antonucci, M. 2010, A\&A, 519, 7

Veilleux, S., Cecil, G., \& Bland-Hawthorn, J. 2005, ARA\&A, 43, 769

Wang, J., \& Wei, J. Y. 2006, ApJ, 648, 158

Wang, J., \& Wei, J. Y. 2008, ApJ, 679, 86

Wang, T., Dai, H., \& Zhou, H. 2008, ApJ, 674, 668 